\title{
Chronische Schmerzen und Arbeitsfähigkeit
}

\section{Roland Schreiber}

Dr. med., Leitender Arzt, multidisziplinäre Schmerzklinik, Spital Bülach

\begin{abstract}
Die Beurteilung der Arbeitsunfähigkeit ist Aufgabe jedes Arztes. Er steht dabei im Spannungsfeld zwischen dem Anspruch des Patienten auf Berücksichtigung seiner Beeinträchtigung und der Forderung von Versicherer und Arbeitgeber nach möglichst rascher Beendigung der Arbeitsunfähigkeit. Die Beeinträchtigung der Arbeitsfähigkeit durch chronische Schmerzen ist ein besonders komplexes Thema.
\end{abstract}

Hinweis

Begriffserläuterungen,

Leitlinien zur

medizinischen

Begutachtung und

die Literatur finden

sich online unter

www.saez.ch $\rightarrow$

Aktuelle Ausgabe oder

$\rightarrow$ Archiv $\rightarrow 2018 \rightarrow 19 / 20$.
Bei einer Arbeitsunfähigkeit unter 3 Monaten oder der realistischen Aussicht auf vollständige Genesung in absehbarer Zeit entstehen keine Probleme. Der behandelnde Arzt stellt das allgemein bekannte Zeugnis aus.

Bei einer Arbeitsunfähigkeit über mehrere Wochen sollte ein detailliertes AUF-Zeugnis für den Arbeitgeber verfasst werden. Hierfür muss eine Arbeitsplatzbeschreibung des Arbeitgebers vorliegen. Dieses Formular kann unter http://www.medforms.ch/Versicherer/ KVG/SIM) heruntergeladen und dem Patienten für seinen Arbeitgeber mitgegeben werden.

Allein schon dieser Prozess macht dem Patienten, aber auch dem Arbeitgeber klar, dass eine Arbeitsunfähigkeit auf den Arbeitsplatz bezogen und gut begründet sein muss. Aber auch, dass der Arbeitnehmer möglichst rasch an seinen Arbeitsplatz zurückzukehren

\section{Résumé}

L'appréciation de l'incapacité à travailler d'un patient est le devoir de chaque médecin. Il se retrouve partagé entre le droit du patient à la reconnaissance de sa condition de malade et les exigences de l'assureur et de l'employeur vis-à-vis d'un arrêt maladie de courte durée et d'une fin de prise en charge rapide. L'atteinte de la capacité à travailler d'une personne à cause de douleurs chroniques est un sujet particulièrement sensible notamment au niveau juridique. Les douleurs chroniques sont une maladie à part entière qui ne peut plus être prise en charge de manière suffisante par les moyens de traitement aigu de la douleur. De même, les douleurs chroniques ne peuvent plus être évaluées selon les concepts des soins médicaux aigus. Pour répondre au concept de traitement bio-psycho-social, il est nécessaire de prendre en compte les critères sociologiques non seulement pour la thérapie mais aussi par rapport à la capacité à travailler du patient. hat und dass der Arbeitgeber verpflichtet ist, den Arbeitsplatz für seinen Mitarbeiter anzupassen.

Von der Arbeitsunfähigkeit ist klar die Erwerbsunfähigkeit zu unterscheiden, wobei es sich um den nach zumutbarer Behandlung und Eingliederung verbleibenden Verlust der Erwerbsmöglichkeiten auf dem ausgeglichenen Arbeitsmarkt handelt. Hierbei werden folgende Kriterien beurteilt:

- Gesundheitsschaden;

- Verlust der Erwerbsmöglichkeiten;

- kausaler Zusammenhang;

- Zumutbarkeit.

Die Beurteilung der Erwerbsfähigkeit ist eine Aufgabe der Versicherungen bzw. der Justiz. Die behandelnden Ärzte dürfen die Erwerbsfähigkeit gesetzlich nicht beurteilen und haben kaum Einfluss auf den Prozess. Da die Entscheidungen bezüglich Reintegrationsmassnahmen, insbesondere Umschulungen oder gar Rentenbeurteilungen, erfahrungsgemäss enorm lange dauern, sind die behandelnden Ärzte häufig gezwungen, die Arbeitsfähigkeit in einem Zeitraum festzulegen, in dem eine Erwerbsfähigkeit längst hätte festgelegt werden sollen. Der Arzt kann aber in dieser Situation lediglich die Arbeitsunfähigkeit im alten Beruf (bzw. am alten Arbeitsplatz) und ein realistisches Belastungsprofil angeben und berufliche Massnahmen anregen. Danach sind ihm die Hände gebunden, und er wartet zusammen mit dem Patienten auf die Entscheidungen der zuständigen Behörden. Er muss aber weiter Arbeitsunfähigkeitszeugnisse ausstellen. Nach der langen Verfahrensdauer hat der soziale Abstieg des Patienten meist stattgefunden, und die Reintegration in die Arbeitswelt gelingt nur noch selten. 
An dieser Stelle muss bemerkt werden, dass die Dauer des Wiedereingliederungs- bzw. Rentenverfahrens einen wesentlichen Einfluss auf die Chronifizierung von Schmerzzuständen hat. Gerade hier wären die Versicherungen wie auch die Justiz in der Pflicht, diese Verfahren möglichst rasch abzuschliessen. Medizinisch kann meist schon nach einigen Monaten der therapeutische Endzustand festgelegt werden. Häufig vergehen aber bis zum Abschluss der Verfahren Jahre. In diesem Zeitraum besteht eine Pattsituation. Der Patient wartet auf die Entscheidung der Behörden. Gleichzeitig sollen die Ärzte in einer Situation, in der der Patient eine Rentenleistung oder eine Wiedereingliederungshilfe bzw. eine Umschulung erwartet, die Reintegration fördern. Dass dies nur sehr selten funktionieren kann, ist offensichtlich.

Chronische Schmerzen sind eine eigenständige Erkrankung und nicht mehr mit den Mitteln der Akutschmerzbehandlung suffizient therapierbar. Sie verstärken sich mit zunehmender Dauer, weshalb eine zeitlich unbeschränkte, bedarfsgerechte Therapie weitergeführt werden muss. Das Ziel ist nicht mehr die Schmerzvernichtung, sondern die Erhaltung des bestmöglichen Schmerz- und Funktionsniveaus des Patienten. Die Therapie orientiert sich am bio-psychosozialen Behandlungsmodell, welches allgemein gefordert und anerkanntermassen wirksam ist. Biologisch sind Medikamente und minimal invasive Methoden neben Trainings- und Physiotherapien zu nennen. Psychologisch steht die Psychoedukation zum Erlernen eines funktionalen Umgangs mit der chronischen Schmerzsituation im Vordergrund. Sozial sind die Reintegration in die Arbeitswelt, die Stabilisierung des sozialen Umfeldes und die finanzielle Sicherheit zu nennen.

Arbeit sollte in meinen Augen als medizinische Massnahme definiert werden und immer in der Behandlung chronischer Schmerzen berücksichtigt werden.

Arbeit sollte in meinen Augen als medizinische Massnahme definiert werden und immer in der Behandlung chronischer Schmerzen berücksichtigt werden. Es sollte sich aber um eine Arbeit handeln, welche dem Anforderungsprofil und den Ressourcen des Patienten gerecht wird. Dies kann bedeuten, dass lediglich ein reduziertes Anforderungsprofil bzw. ein reduziertes Arbeitspensum möglich ist. Wird der Patient überfordert, wird es erst zur Zunahme von medizinischen Behandlungen, dann zu Arbeitsausfällen und schliesslich zum Arbeitsplatzverlust kommen, leider eine häufige Erfahrung, nicht nur im schmerzmedizinischen Alltag.

\section{Chronische Schmerzen sind auch nicht mehr mit den} Konzepten der Akutmedizin beurteilbar. Aus diesem Grunde hat sich für dieses komplexe Spezialgebiet eine Zusatzausbildung etabliert. Es gibt unterdessen auch in der Schweiz zwei Weiterbildungstitel (Schmerzspezialist ${ }^{\circledR}$ SGSS und Fähigkeitsausweis Interventionelle Schmerztherapie SSIPM). Ein erster Schritt wäre, bei der Begutachtung von chronischen Schmerzzuständen eine entsprechende Fachperson, d.h. einen Schmerzmediziner mit langjähriger, therapeutischer Erfahrung, hinzuzuziehen.

\section{Wir haben es auch in der Schmerzmedizin mit} einigen hartnäckigen, veralteten Konzepten zu tun.

Wir haben es jedoch in der Schmerzmedizin mit einigen hartnäckigen, veralteten Konzepten zu tun, z.B. der Trennung von Körper, Psyche und Umwelt. Dieses Konzept, welches auf Descartes zurückgeht, ist längst durch die wissenschaftlichen Erkenntnisse der Hirnforschung widerlegt worden. Zahlreiche Forschungen zeigen uns, dass im Hirn bei körperlichem Training und mentaler Aktivität die gleichen Areale aktiv sind. Eine Erkenntnis, welche im Sport längst umgesetzt wurde, die in der Medizin jedoch immer noch als Kuriosum gehandelt wird. Ferner ist auch bekannt, dass die Schmerzmatrix bei sozialer Ablehnung genauso aktiviert wird wie bei körperlichem Schmerz. Diesen Tatsachen soll in der Schmerzmedizin durch die Arbeit mit dem bio-psycho-sozialen Modell Rechnung getragen werden. Für die sozialen Belange sind jedoch weder die Krankenkassen noch die Unfall- und Taggeldversicherungen oder die IV zuständig. Wie dargelegt, macht das aus medizinischer Sicht keinen Sinn. In der praktischen Tätigkeit entsteht so eine Lücke, die kaum geschlossen werden kann.

Die Medizin soll verbinden, was die Justiz dann wieder trennt!

Die Tatsachen aus der Rechtsprechung müssen akzeptiert werden, was aber die Arbeit mit chronischen Schmerzpatienten nicht erleichtert. Häufig ist diese Trennung für den Patienten sogar fatal.

Man hört noch immer, dass es Menschen gibt, die mit Schmerzen arbeiten können. Deshalb seien Schmerzen per se kein Grund für eine längerdauernde Arbeitsunfähigkeit (Argument der Überwindbarkeit). Dies ist in der Beurteilung der Beeinträchtigung durch Schmerzen keine Hilfe, wie schon die vielen Gerichtsprozesse zeigen (z.B. Revision des Bundegerichtsbeschlusses [9c_492/2014] bezüglich der Überwindbarkeit von Schmerzen vom Juni 2015). 
Chronische Schmerzen können, wie die schmerzmedizinische Praxis zeigt, durchaus zu alltagsrelevanten Beeinträchtigungen führen. Das Ausmass derselben setzt sich meiner Meinung nach aus folgenden drei Komponenten zusammen:

- medizinischer Einschränkung;

- Einschränkung durch Verlangsamung (schmerzbedingt, kognitiv, medikamentös);

- Einschränkung durch den zeitlichen Aufwand an Therapien zur Erhaltung des Funktionsniveaus.

Das Ausmass der Beeinträchtigung und insbesondere die Beurteilung der Zumutbarkeit können nicht beurteilt werden, ohne die subjektiven Krankheitskonzepte des Patienten ebenso wie seine Copingstrategien in Rechnung zu stellen.

Bei den subjektiven Krankheitskonzepten können drei Aspekte unterschieden werden:

- Kognitive Aspekte: beeinflusst u.a. durch:

- Herkunftsland/kulturellen Hintergrund

- Schulbildung

- Informationsstand über die eigene Erkrankung

- Lokalisation der Krankheitskontrolle: intern, zufällig bzw. schicksalhaft, extern durch den Arzt oder eine Institution

- subjektive Beurteilung des Schweregrades der Symptome und die subjektive Prognose

- Emotionale Aspekte wie:

- Scham

- Schuldzuweisung oder Überzeugung, bestraft zu werden

- Anspruchshaltung auf eine Entschädigung oder dass andere das Problem lösen

- Akzeptanz

- Verleugnung

- Aggression

- Depression

- Motivationale Aspekte wie:

- Überzeugung, selbst etwas zur Verbesserung der Situation beitragen zu können

- Überzeugung, vom Schicksal geschlagen zu sein

- Überzeugung, dass alles sowieso sinnlos ist

Bei den Copingstrategien erfolgt die Beurteilung am einfachsten durch den Bewältigungsstil:

- verleugnend

- sinnsuchend

- ablehnend

- Verantwortung anderen zuweisend

Korrespondenz:

Roland Schreiber

Häldeliweg 35

CH-8044 Zürich

schreiberry[at]bluewin.ch
Besteht ein dysfunktionales Coping, sollte das Potential zur Erlernung von funktionalen Copingstrategien berücksichtigt werden. Dieses ist abhängig von den kognitiven Fähigkeiten (mentale Prozesse, welche Informationen verarbeiten, die Fähigkeit, Neues zu lernen und Wissen zu verarbeiten) und der Motivation, etwas zu ändern.

Die Beurteilung der kognitiven Fähigkeiten kann z.B. durch die kognitiven Aspekte des Krankheitskonzeptes (siehe oben), Sprachkenntnisse in Bezug zur Schulbildung und hauptsächlichen Kommunikation am Arbeitsplatz, Tests (Zahlenverbindungstest, Turm von Hanoi) erfolgen.

Über die Motivation geben z.B. Arbeitsversuche, laufende Renten- bzw. Versicherungsverfahren und der Tagesablauf bzw. die Tagesstruktur gute Hinweise.

Selten besteht noch ein Potenzial für eine Verbesserung des Coping bei:

- etablierter Chronifizierung

- mangelnden kognitiven Fähigkeiten

- chronifiziertem sozialem Abstieg (z.B. Langzeitarbeitslosigkeit)

- etabliertem Arrangement in der momentanen Situation

- fehlendem Vertrauen in die eigenen Fähigkeiten

- durchgeführten Schulungen bzw. Therapien ohne Veränderung der Situation

- Erwartung einer Rente oder finanziellen Entschädigung

- Stigmatisierung durch andere und sich selbst

Die Beurteilung der Arbeitsunfähigkeit und der Beeinträchtigung durch chronische Schmerzen, welche ich im «Arbeitspapier Beeinträchtigung durch chronische Schmerzen" vorschlage, erfolgt mit dem bio-psycho-sozialen Ansatz. Dieses Arbeitspapier kann auf der Homepage praxis-schreiber.ch heruntergeladen werden.

Dass soziale Aspekte bei chronischen Erkrankungen versicherungsrechtlich nicht anerkannt werden, entbindet den behandelnden Arzt nicht davon, diese mitzuberücksichtigen. Ich kann nur nochmals betonen, dass soziale Aspekte bei chronischen Erkrankungen medizinisch relevant sind und in die Therapie miteinbezogen werden müssen.

\section{Disclosure}

keine Interessenverbindungen 
Für die zahlreichen Begriffsdefinitionen verweise ich auf die Artikel der SIM Swiss Insurance Medicine:

- https://www.swiss-insurance-medicine.ch/tl files/firstTheme/ PDF\%2ODateien\%2Oab\%202015/4\%2OFachwissen\%2Onachschlagen/Arbeitsunfaehigkeit\%2O(Zeugnisse\%20verlinkt\%20mit\%20 medforms.ch)/SIM\%2OAUF\%2OBroschuere_2013_D.pdf Retrieved Mai 17, 2017

- https://www.swiss-insurance-medicine.ch/tl files/firstTheme/ PDF\%2ODateien\%2Oab\%202015/4\%2OFachwissen\%2Onachschlagen/Arbeitsunfaehigkeit\%2O(Zeugnisse\%20verlinkt\%20mit\%20 medforms.ch)/SIM\%2OZumutbare\%20Arbeitstaetigkeit\%20 Broschuere_2013_D.pdf Retrieved Mai 17, 2017

Leitlinien medizinische Begutachtung:

- https://www.swiss-insurance-medicine.ch/tl_files/firstTheme/ PDF\%2ODateien\%20ab\%202015/4\%20Fachwissen\%20nachschlagen/Medizinische\%20Gutachten/SIM_Med_Begutachtung Schweiz_2017_d.pdf

- https://www.swiss-insurance-medicine.ch/tl files/firstTheme/ PDF\%2ODateien\%2Oab\%202015/4\%20Fachwissen\%2Onachschlagen/Leitlinien/Leitlinien_neuropsychologische_Begutachtung. pdf

- http://www.psychiatrie.ch/sgpp/fachleute-und-kommissionen/

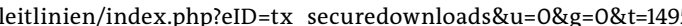
086182\&hash=089876b1ae2eae1b2478d052950cacd302cd06fa\& file=/fileadmin/SGPP/user_upload/Fachleute/Empfehlungen Qualitaet/D_Qualitaetsleitlinien_fuer_versicherungspsychiatrische_Gutachten_20.10.2016.pdf

- http://www.rheuma-net.ch/download/Content_attachments/ FileBaseDoc/Leitlinien-fur-die-rheumatologische-Begutach tung-12-2016.pdf

- http://www.swissorthopaedics.ch/images/content/Empfehlungen/Begutachtung_2_2017/D-LeitlinienGutachten-2.2017.pdf Retrieved Mai 21, 2017

Literatur:

- Altier A, et al. (2006). DEGAM - Versorgung Chronisch Kranker - Positionspapier - Entwurf V1, Kap. 2.2. Chronisch krank sein subjektiv, www.allgemeinmedizin.uni-frankfurt.de/.../2_2_DEGAM Altina_Subjektivitaet.pdf, Retrieved Mai 25, 2017

- Brulhart V, et al. (2007). Zur Arbeitsunfähigkeit verurteilt? Basel: swissprofessionalmedia AG, Medical Tribune, www.svv.ch/sites/ default/files/.../file/Medical-Tribune_Handout-zum-Seminar.pdf Retrieved Mai 19, 2017
- Büchi S/Buddeberg C. Subjektive Krankheitskonzepte-Krankheitsbewältigung. In: Psychosoziale Medizin. 409-30. Berlin Heidelberg: Springer Verlag; 2004.

- Oliveri M, et al. Grundsätze der ärztlichen Beurteilung der Zumutbarkeit und Arbeitsfähigkeit. Teil 1. Schweiz Med Forum. 2006;6: 420-31.

- Oliveri M, et al. Evaluation der funktionellen Leistungsfähigkeit EFL nach Susan Isernhagen. SUVA-Medizinische Mitteilungen. Suva Luzern. 1996;69:15-27.

- Oliveri M. Was sollen wir messen: Schmerz oder Funktion? Die Evaluation der funktionellen Leistungsfähigkeit als Mittel für die Beurteilung der Arbeitsfähigkeit. In: Schmerz und Arbeitsfähigkeit (Eds. Schaffhauser R, Schlauri F). Universität St. Gallen, Institut für Rechtswissenschaft und Rechtspraxis, 2003; 390-407.

- Petrie KJ/Weinman JA (1997). Perceptions of health and illness. Amsterdam: Harwood Academic Publishers.

- Salewski C (2000). Subjektive Krankheitskonzepte. Heidelberg: Spektrum Akademischer Verlag, http://www.spektrum.de/lexikon/psychologie/subjektive-krankheitskonzepte/15028, Retrieved Juni 1, 2017

- Schonstein E, Denny DT, Keating J, Koes BW. Work conditioning, work hardening and functional restoration for workers with back and neck pain. Cochrane Database Syst Rev. 2003;(1):CD001822.

- Schreiber R. Gesichter des Schmerzes - Möglichkeiten und Horizonte der Schmerzmedizin. Zürich: Careum Verlag; 2017.

- Schweizerische Gesellschaft der Vertrauens- und Versicherungsärzte. Beurteilung des Grades der Arbeitsunfähigkeit, https:// www.vertrauensaerzte.ch/manual/4/aufarztzeugnis/beurteilunggrad, Retrieved Mai 17, 2017

- Waddell G. The Back Pain Revolution. Churchill Livingstone, 2. Auflage, 2005.

- World Health Organization (2003). ICF Checklist revSep2003, http://www.who.int/classifications/icf/training/icfchecklist.pdf, Retrieved Mai 5, 2017

- World Health Organization. How to use the ICF: A practical manua for using the International Classification of Functioning, Disability and Health (ICF). Exposure draft for comment. Geneva: WHO; October 2013 